\title{
Zebrafish and Medaka: new model organisms for modern biomedical research
}

\author{
Cheng-Yung Lin, Cheng-Yi Chiang and Huai-Jen Tsai
}

\begin{abstract}
Although they are primitive vertebrates, zebrafish (Danio rerio) and medaka (Oryzias latipes) have surpassed other animals as the most used model organisms based on their many advantages. Studies on gene expression patterns, regulatory cis-elements identification, and gene functions can be facilitated by using zebrafish embryos via a number of techniques, including transgenesis, in vivo transient assay, overexpression by injection of mRNAs, knockdown by injection of morpholino oligonucleotides, knockout and gene editing by CRISPR/Cas9 system and mutagenesis. In addition, transgenic lines of model fish harboring a tissue-specific reporter have become a powerful tool for the study of biological sciences, since it is possible to visualize the dynamic expression of a specific gene in the transparent embryos. In particular, some transgenic fish lines and mutants display defective phenotypes similar to those of human diseases. Therefore, a wide variety of fish model not only sheds light on the molecular mechanisms underlying disease pathogenesis in vivo but also provides a living platform for high-throughput screening of drug candidates. Interestingly, transgenic model fish lines can also be applied as biosensors to detect environmental pollutants, and even as pet fish to display beautiful fluorescent colors. Therefore, transgenic model fish possess a broad spectrum of applications in modern biomedical research, as exampled in the following review.
\end{abstract}

Keywords: Disease model organism, Developmental biology, Gene regulation, in vivo expression, Transgenic fish

\section{Background}

Although zebrafish (Danio rerio) and medaka (Oryzias latipes) are primitive vertebrates, they have several advantages over other model animals. For example, they are fecund and light can control their ovulation. Spawning takes place frequently and no limitation in their spawning season. Microinjection of fertilized eggs is easily accessible and relatively cheap. Their embryos are transparent, making it easy to monitor the dynamic gene expression in various tissues and organs in vivo without the need to sacrifice the experimental subjects. Their genome sizes are approximately 20 to $40 \%$ of the mammalian genome, making them the only vertebrates available for large-scale mutagenesis. Their maturation time takes only $2 \sim 3$ months, which is relatively less laborious and time-saving for generating transgenic lines. In addition, many routine techniques of molecular biology and genetics, including knock-in, knockdown and

\footnotetext{
* Correspondence: hjtsai@ntu.edu.tw

Graduate Institute of Biomedical Sciences, Mackay Medical College, No.46, Section 3, Zhongzheng Rd., Sanzhi Dist., New Taipei City 252, Taiwan
}

knockout, are well developed in the model fish. Therefore, zebrafish and medaka are new excellent animal systems for the study of vertebrate-specific biology in vivo.

\section{Review \\ Germline transmission}

The F0 transgenic line can be established once the exogenous gene can be successfully transferred to the embryos, followed by stable germline transmission of the transgene to the F1 generation. Generally, around 10-20\% of treated embryos have a chance to achieve germline transmission [1]. It has been reported that a foreign gene flanked with inverted terminal repeats of adeno-associated virus can be used to enhance the ubiquitous expression and stable transmission of transgene in model fish [2]. Meanwhile, transgenesis can be facilitated by using the Tol2 transposon derived from medaka [3]. Transposase catalyzes transposition of a transgene flanked with the Tol2 sequence [4]. The efficiency of Tol-2-mediated germline transmission could range from 50 to $70 \%$ of injected embryos $[4,5]$. 
A cutting-edge technique has taken the study of fish gene transfer to new horizons, such as knockout zebrafish by the transcription activator-like effector nuclease (TALEN) system and the clustered regularly interspaced short palindromic repeats (CRISPR) combined with CRISPR-associated proteins 9 (Cas9) [6, 7]. The TALEN system involves the DNA recognition domain of transcription activator-like effectors (TALEs) and a nuclease domain for generation of nicks on DNA sequences. The CRISPR/Cas9 system directed by a synthetic single guide RNA can induce targeted genetic knockout in zebrafish. The main difference between these two systems is based on their recognition mechanisms. Unlike the TALEs applied in the TALEN system, the CRISPR/Cas9 system recognizes its target DNA fragment by the complementary non-coding RNA. The development of the TALEN and CRISPR/Cas9 systems provides new genomic editing approaches for establishing genetic knockout fish lines [8].

\section{Transgenic fluorescent fish}

The fluorescence protein gene (FPG) has been widely applied as a reporter gene in studies of the transgene expression by direct visualization under fluorescent microscopy in vivo [9]. Many transgenic model fish lines harbor an FPG driven by various tissue-specific promoters, including the erythroid-specific GATA promoter [10], muscle-specific $\alpha$-actin promoter [11], rod-specific rhodopsin promoter [12], neuron-specific isl-1 promoter [13], pancreas-specific $p d x-1$ and insulin promoters [14], myocardium-specific $\mathrm{cmlc} 2$ promoter [15], liver-specific L-FABP promoter [16], bone-specific col10a1 promoter [17], macrophage-specific mfap4 promoter [18], and germ cell-specific vasa promoter [19]. Using medaka $\beta$-actin promoter, Tsai's lab generated a transgenic line of medaka displaying green FP ubiquitously around the whole fish from F0 through F2 generations in a Mendelian inheritance manner [20]. This is known as the first transgenic line of glowing pet fish, which was reported by Science [21] and Far Eastern Economic Review [22] and honored to be selected as among "The Coolest Inventions of 2003" by TIME [23]. The DNA sequences of the aforementioned promoters ranging from 0.5 to $6.5 \mathrm{~kb}$ are sufficient to drive the FPG reporter to mimic the tissue-specific expression of endogenous gene.

However, some genes require a longer regulatory DNA sequence, such as more than $20 \mathrm{~kb}$, to fully recapitulate the characteristic expression profiles of endogenous genes. In that case, bacterial artificial chromosome (BAC) and phage P1-dereived artificial chromosome (PAC) have been commonly used for this purpose [24]. For example, the zebrafish rag1 gene, flanked with PAC DNA containing $80 \mathrm{~kb}$ at the $5^{\prime}$ upstream and $40 \mathrm{~kb}$ at the 3' downstream, can be expressed specifically in lymphoid cells. Instead of using the tedious Chi-site dependent approach, Jessen et al. reported a two-step method to construct a BAC clone [25]. Employing this protocol, Chen et al. constructed a BAC clone containing the upstream $150 \mathrm{~kb}$ range of zebrafish myf5 and generated a transgenic line $\operatorname{Tg}(m y f 5: g f p)$ [26]. This transgenic line is able to recapitulate the somite-specific and stagedependent expression of the endogenous myf5 at an early developmental stage. In summary, all the above transgenic lines should be very useful materials for studying both gene regulation and cell development.

\section{New materials for developmental, biological and biomedical studies}

\section{A) Developmental biology}

\section{Heart development}

Zebrafish is particularly useful for studying heart development for the following reasons: (a) Zebrafish have a primitive form of the heart, which is completely developed within $48 \mathrm{~h}$ post-fertilization (hpf). (b) The cardiac development can be easily observed in the transgenic line possessing a FP-tagged heart. (c) The zebrafish embryos with a defective cardiovascular system can still keep on growing by acquiring oxygen diffused from water. (d) Discovery of genes involved in heart development can be facilitated by a simple haploid mutation method [27]. For example, using the zebrafish jekyll mutant, which has defective heart valves, Walsh and Stainier discovered that UDPglucose dehydrogenase is required for zebrafish embryos to develop normal cardiac valves [28].

Tsai's lab is the first group to generate a transgenic zebrafish line that possesses a GFP-tagged heart [15]. This line was established from zebrafish embryos introduced with an expression construct, in which the GFP reporter is driven by an upstream control region of zebrafish cardiac myosin light chain 2 gene $(\mathrm{cmlc} 2)$. Using this transgenic line, Raya et al. found that the Notch signaling pathway is activated is during the regenerative response [29]. Shu et al. reported that $\mathrm{Na}$, K-ATPase $\alpha 1 \mathrm{~B} 1$ and $\alpha 2$ isoforms have distinct roles in the patterning of zebrafish heart [30]. This transgenic line should also be useful for studying the dynamic movement and cell fate of cardiac primordial cells. For example, Forouhar et al. proposed a hydro-impedance pump model for the embryonic heart tubes of zebrafish [31]. A 4D dynamic image of cardiac development has been developed [32]. Furthermore, Hami et al. reported that a second heart field is required during cardiac development [33]. Thus, recently, Nevis et al. stated that $t b \times 1$ plays a function for proliferation of the second heart field, and the zebrafish tbx1-null mutant resemble the heart defects in DiGeorge syndrome [34].

Thus, the expression pattern of heart-specific genes could be analyzed based on heart progenitor cells collected 
in this transgenic line. The analysis of gene or protein expression dynamics at different developmental stages could also be conducted. Furthermore, this transgenic fish is a potential platform for detecting chemicals, drugs and environmental pollutants affecting heart development, as detailed in following section.

\section{Muscle development}

In vivo transient assay of the injected DNA fragments in model fish embryos is a simple yet effective way to analyze the function of regulatory cis-elements. For example, Myf5, one of myogenesis regulatory factors (MRF), plays key roles in the specification and differentiation of muscle primordial cells during myogenesis. The expression of $m y f 5$ is somite-specific and stage-dependent, and its activation and repression are delicately orchestrated. Using in vivo transient assay, Chen et al. found that a novel ciselement located at $-82 /-62$ is essential for somite-specific expression of myf5 [35]. Lee et al. revealed that this $-82 /-$ 62 cis-element is specifically bound by Forkhead box D3, and proposed that somite development is regulated by the Pax3-FoxD3-Myf5 axis [36]. Besides FoxD3, FoxD5, another protein in the Forkhead box family, is necessary for maintaining the anterior-posterior polarity of somite cells in mesenchymal-epithelial transition [37]. The expression of FoxD5 is regulated by Fgf signaling in anterior presomitic mesoderm (PSM), which indicates that Fgf-
FoxD5-Mesp signaling takes place in somitogenesis [37]. Furthermore, analysis of the loci of adjacent mrf4 and myf5 revealed the complicated regulation mechanism of the MRF genes. It was also found that the biological function of Mrf4 is related to myofibril alignment, motor axon growth, and organization of axonal membrane [38].

The molecular mechanism that underlies the repression of $m y f 5$ has also been reported. For example (Fig. 1a), a strong repressive element of zebrafish myf5 was found within intron I $(+502 /+835)$ [39]. This repressive element is modulated by a novel intronic microRNA, termed miRIn300 or miR-3906 [40]. When myf5 transcripts reach the highest level after specification, the accumulated miR3906 starts to reduce the transcription of myf5 through silencing the positive factor Dickkopf-related protein 3 (Dkk3r or Dkk3a) for the myf5 promoter [41]. Itga6b is a receptor of secretory Dkk3a and that interaction between Itg $\alpha 6 \mathrm{~b}$ and Dkk3a is required to drive the downstream signal transduction which regulates myf5 promoter activity in somite during embryogenesis of zebrafish [42]. Dkk3a regulates p38a phosphorylation to maintain Smad4 stability, which in turn enables the formation of the Smad2/3a/4 complex required for the activation of the myf5 promoter [43]. However, when myf5 transcripts are reduced at the later differentiation stage, miR-3906 is able to be transcribed by its own promoter [40] (Fig. 1b). Furthermore, increased expression of miR-3906

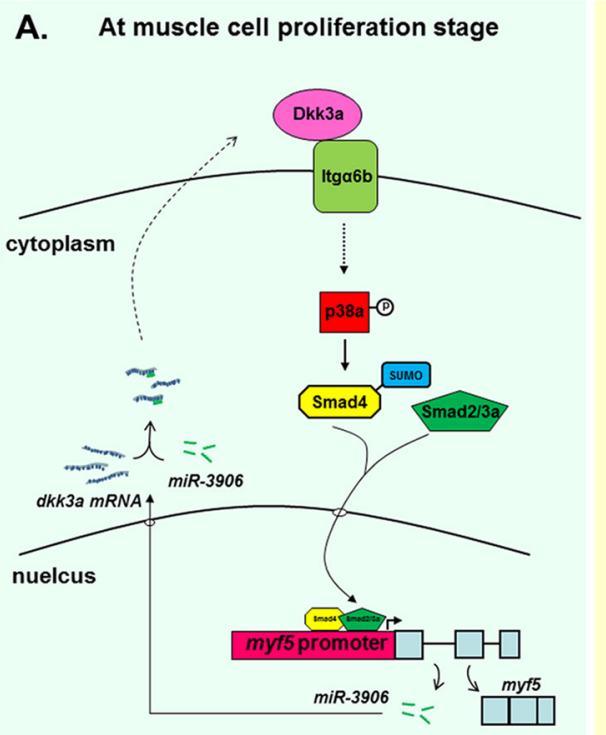

B. At muscle cell differentiation stage

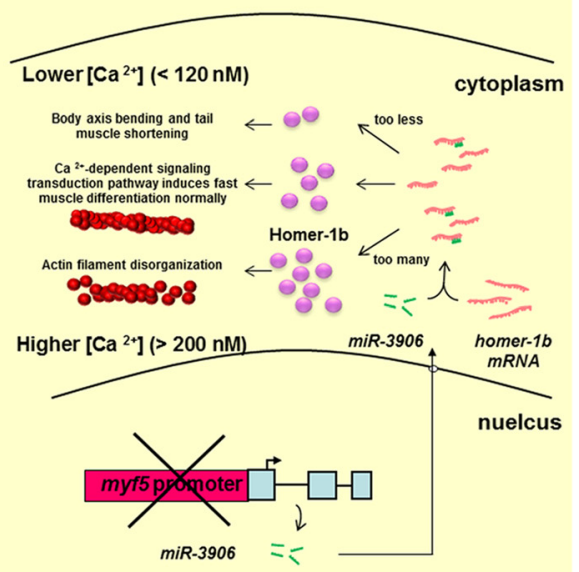

Fig. 1 miR-3906 silences different target genes at different muscle developmental stages. a At the early muscle development, Dkk3a interacts with its receptor Itga6b, resulting in the phosphorylation of p38a and the formation of the Smad2/3a/4 complex, which in turn, activates the myf5 promoter activity. When myf5 is highly transcribed, the intronic miR-3906 suppresses the transcription of myf5 through silencing the Dkk3a [39, 41-43]. b At the late muscle development, miR-3906 starts transcription at its own promoter and switches to silence homerib to control the homeostasis of intracellular calcium concentration $\left(\left[\mathrm{Ca}^{2+}\right]_{\mathrm{i}}\right)$ in fast muscle cells [40]. Either miR-3906-knockdown or homer16 -overexpression causes the increase of Homer-1b protein, resulting in an enhanced level of $\left[\mathrm{Ca}^{2+}\right]_{\text {i, }}$ which in turn, disrupts sarcomeric actin filament organization. In contrast, either miR-3906-overexpression or homer-1b-knockdown causes the decrease of Homer-1b, resulting in a reduced $\left[\mathrm{Ca}^{2+}\right]_{i}$ and thus a defective muscle phenotype [40] 
controls the intracellular concentration of $\mathrm{Ca}^{2+}\left(\left[\mathrm{Ca}^{2+}\right]_{\mathrm{i}}\right)$ in fast muscle cells through subtly reducing homer-1b expression. The homeostasis of $\left[\mathrm{Ca}^{2+}\right]_{\mathrm{i}}$ is required during differentiation to help maintain normal muscle development [40]. Nevertheless, it remains to be investigated how miR-3906 switches its target gene at different developmental stages.

Apart from the regulation of somitogenesis, Myf5 is also involved in craniofacial muscle development. The functions of Myf5 in cranial muscles and cartilage development are independent of Myod, suggesting that Myf5 and Myod are not redundant. Thus, three possible pathways could be associated with the molecular regulation between Myf5 and Myod: (i) Myf5 alone is capable of initiating myogenesis, (ii) Myod initiates muscle primordia, which is subdivided from the myf5-positive core, and (iii) Myod alone, but not Myf5, modulates the development of muscle primordia [44]. Furthermore, the sixla gene was found to play an important role in the interaction between Myf5 and Myod [45]. In cartilage development, myf5 is expressed in the paraxial mesoderm at the gastrulation stage. Myf5 plays a role in mesoderm fate determination by maintaining the expression of $f g f 3 / 8$, which in turn, promotes differentiation from neural crest cells to craniofacial cartilage [46]. This research on myf5 not only reveals that it has different functions between craniofacial muscle development and somitogenesis, but it also opens up a new study field for understanding craniofacial muscle development.

Hinits et al. reported no phenotype both in myf5knockdown embryos and myf5-null mutant, suggesting that Myf5 is rather redundant in somitogenesis of zebrafish [47]. However, it is hard to reasonably explain why these embryos and mutant are all lethal and can't grow to adulthood. On the other hand, Lin et al. reported an observable defective phenotype in $m y f 5$-knockdown embryos, and claimed that the concentration of myf5-MO they used can inhibit maternal myf5 mRNA translation [46]. This discrepancy might attribute the effectiveness of MO used or the different phenotypes between the knockdown embryos and knockout mutant in this case.

\section{Ocular and nerve development}

The retina-specific expression of the carp rhodopsin gene is controlled by two upstream regulatory DNA ciselements [48]. One is located at -63 to -75 , which is the carp neural retina leucine zipper response-like element; the other is located at -46 to -52 , which is a carpspecific element crucial to reporter gene expression in medaka retinae. Intriguingly, immediate activation of early growth response transcriptional regulator Egr1 could result in the incomplete differentiation of retina and lens, leading to microphthalmos [49]. Another important factor for ocular development is the ADPribosylation factor-like 6 interacting protein 1 (Arl6ip1). Loss of Arl6ip1 function leads to the absence of retinal neurons, disorganized retinal layers and smaller optic cups [50]. Upon losing Arl6ip1, retinal progenitors continued to express cyclin D1, but not shh or p57kip2, suggesting that eye progenitor cells remained at the early progenitor stage, and could not exit the cell cycle to undergo differentiation [51]. Additionally, it has been reported that Arl6ip1 is essential for specification of neural crest derivatives, but not neural crest induction. $\mathrm{Tu}$ et al. found that Arl6ip1 mutation causes abnormal neural crest derivative tissues as well as reduced expression of neural crest specifier genes, such as foxd3, snail1b and sox10, indicating that Arl6ip1 is involved in specification, but not induction, of neural crest cells [52]. Furthermore, they found that Arl6ip1 could play an important role in the migration of neural crest cells because in the arl6ip1-knockdown embryos, crestinand sox10-expressing neural crest cells failed to migrate ventrally from neural tube into trunk. More recently, Lin et al. found that Ras-related nuclear (Ran) protein is conjugated with Arl6ip1, and proposed that Ran protein associates with Arl6ip1 to regulate the development of retinae [53].

To date, no in vivo model system has been established to identify cells in the CNS that can specifically respond with regeneration after stresses, and, even if identified, no method is in place to trace these responsive cells and further identify their cell fates during hypoxic regeneration. To address these issues, Lee et al. generated a transgenic zebrafish line $h u O R F Z$, which harbors the upstream open reading frame (uORF) from human CCAAT/enhancer-binding protein homologous protein gene (chop), fused with the GFP reporter and driven by a cytomegalovirus promoter [54]. After huORFZ embryos were treated with heat-shock or under hypoxia, the GFP signal was exclusively expressed in the CNS, resulting from impeding the huORF ${ }^{\text {chop }}$-mediated translation inhibition [54]. Interestingly, Zeng et al. found that GFP-(+) cells in spinal cord respond to stress, survive after stress and differentiate into neurons during regeneration (Chih-Wei Zeng, Yasuhiro Kamei and Huai-Jen Tsai, unpublished data).

\section{MicroRNAs and development}

MicroRNAs (miRNAs) are endogenous single-stranded RNA molecules of 19-30 nucleotides (nt) that repress or activate the translation of their target genes through canonical seed- and non-canonical centered miRNA binding sites. The known mechanisms involved in miRNAs-mediated gene silencing are decay of mRNAs and blockage of translation [55-57]. Probably the expression of $30 \sim 50 \%$ of human genes is regulated by 
miRNAs [58, 59]. Therefore, to understand gene and function in cells or embryos, it is important to exactly know the target gene(s) of a specific miRNA at different phase of cells or at particular stages of developing embryos.

Instead of using a bioinformatic approach, the Tsai's lab developed the Labeled miRNA Pull-down (LAMP) assay system, which is a simple but effective method to search for the candidate target gene(s) of a specific miRNA under investigation [60]. LAMP assay system yields fewer falsepositive results than a bioinformatic approach. Taking advantage of LAMP, scientists discovered that miR-3906 silences different target genes at different developmental stages, e.g., at early stage, miR-3906 targets $d k k 3 a$ [41], while at late stage, it targets homer-1b [40] (Fig. 1).

In another example (Fig. 2), $m i R-1$ and $m i R-206$ are two muscle-specific microRNAs sharing the same seed sequences. They are able to modulate the expression of vascular endothelial growth factor Aa (VegfAa) and serve as cross-tissue signaling regulators between muscle and vessels. Since $m i R-1$ and $m i R-206$ share identical seed sequences, Stahlhut et al. demonstrated that they can silence the same target gene, such as vegfaa, and considered them as a single cross-tissue regulator termed as $m i R-1 / 206$ [61]. $m i R-1 / 206$ reduces the level of VegfAa, resulting in the inhibition of the angiogenic signaling [61]. Surprisingly, using the LAMP assay system, Lin et al. reported that the target genes for $m i R-1$ and $m i R-206$ are different [62]. While miR-206 targets vegfaa, miR-1 targets seryl-tRNA synthetase gene (sars). SARS is a negative regulator of VegfAa. Although both miR-1 and miR-206 have identical seed sequences, the sars-3'UTRs of zebrafish, human and mouse origins can be recognized only by miR-1 in zebrafish embryos and mammalian cell lines (HEK-293 T and C2C12), but not by miR-206 [62]. Conversely, the vegfaa-3'UTR is targeted by $m i R-206$, but not by $m i R-1$. Therefore, Lin et al. concluded that miR-1 and miR-206 are actually two distinct regulators and play opposing roles in zebrafish angiogenesis. The miR-1/SARS/VegfAa pathway promotes embryonic angiogenesis by indirectly controlling VegfAa, while miR-206/VegfAa pathway plays an anti-angiogenic role by directly reducing VegfAa. Interestingly, they also found that the miR-1/SARS/VegfAa pathway increasingly affects embryonic angiogenesis at late developmental stages in somitic cells [62]. It remains to be studied how miR-1 increases in abundance at late stage.

\section{miRNAs in regeneration}

Different from mammals, zebrafish have the ability to regenerate injured parts in the CNS. Many miRNAs have been found in the CNS. Since miRNAs are involved in many aspects of development and homeostatic pathways, they usually play important roles in regeneration [63]. It has been shown that several miRNAs have prominent

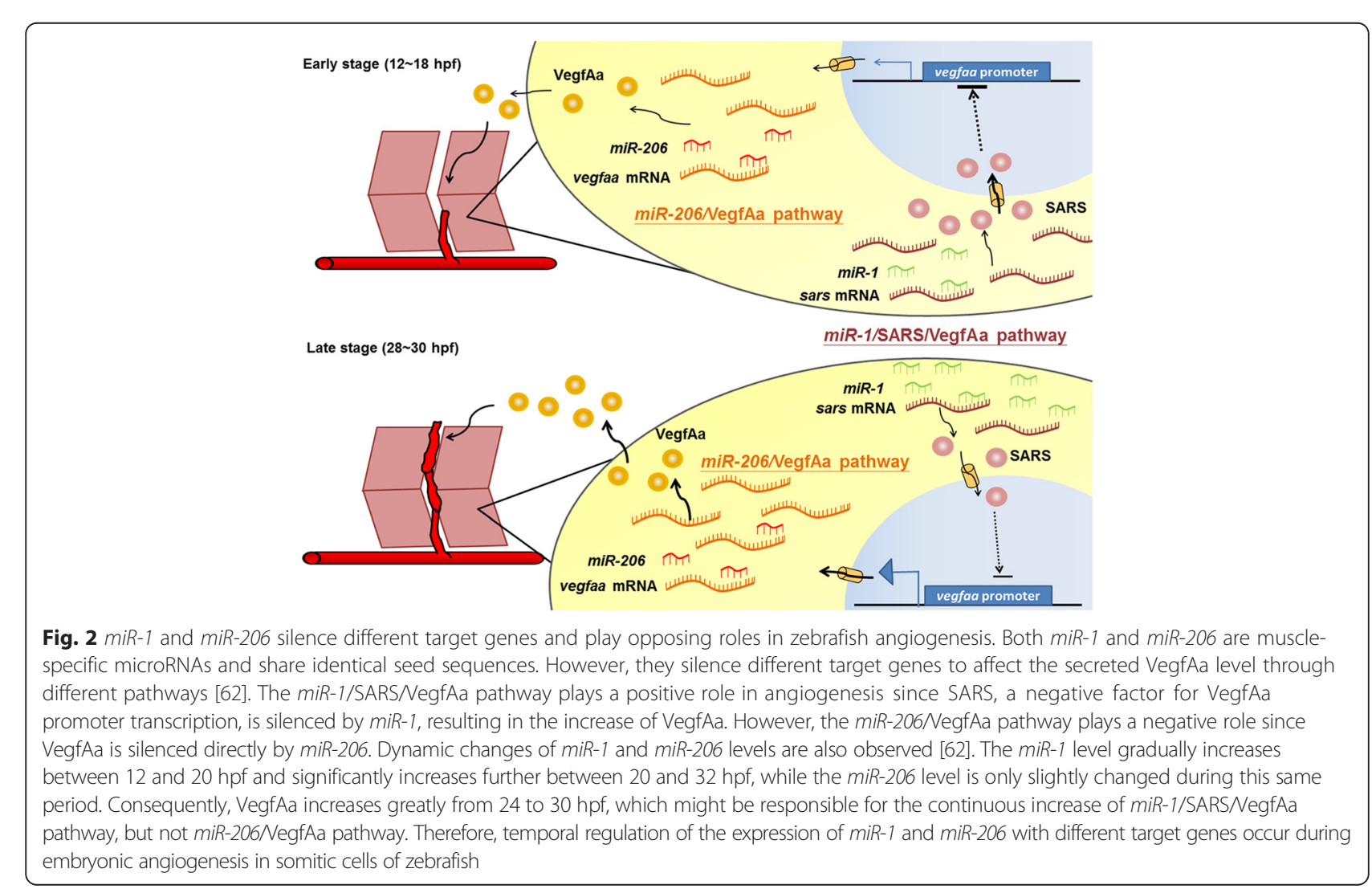


functions in regulating the regeneration process. For example, $m i R-210$ promotes spinal cord repair by enhancing angiogenesis [64], and the miR-15 family represses proliferation in the adult mouse heart [65]. Furthermore, miRNAs $m i R-29 b$ and $m i R-223$ are identified following optic nerve crush. By gene ontology analysis, $m i R-29 b$ and $m i R$ 223 are found to regulate genes, including eva1a, layna, nefmb, ina, si:ch211-51a6.2, smoc1, and sb:cb252. These genes are involved in cell survival or apoptosis, indicating that these two miRNAs are potential regulators of optic nerve regeneration [66].

\section{Hematopoiesis}

Although the main hematopoietic sites in zebrafish differ from those in mammals, both zebrafish and mammals share all major blood cell types that arise from common hematopoietic lineages [67]. Moreover, many genes and signaling pathways involved in hematopoiesis are conserved among mammals and zebrafish. For example, scl, one of the first transcription factors expressed in early hematopoietic cells, is evolutionarily conserved. During definitive hematopoiesis, runx1 marks hematopoietic stem cells (HSCs) in both mouse and fish. Additionally, in differentiated populations, gata1, the erythroid lineage regulator, pu. 1 and clebp, the myeloid lineage regulators, and ikaros, a mark of the lymphoid population, are in accordance with the hematopoietic hierarchy in zebrafish and mammals [68]. Thus, the findings with respect to zebrafish blood development could be applied to mammalian system.

Genetic screening in zebrafish has generated many blood-related mutants that help researchers understand hematopoietic genes and their functions [69]. For example, the spadetail mutant carrying a mutated tbx16 exhibits defective mesoderm-derived tissues, including blood. This mutant displays the decrease levels tal1, lmo2, gata2, fli1 and gata1 in the posterior lateral mesoderm, indicating the important role of $t b x 16$ during hemangioblast regulation [70]. Chemical screening in zebrafish using biologically active compounds is also a powerful approach to identify factors that regulate HSCs. For example, it is well known that prostaglandin (PG) E2 increases the induction of stem cells in the aorta-gonad-mesonephros region of zebrafish, as demonstrated by increasing expressions of runx 1 and $c m y b$, which, in turn, increases engraftment of murine marrow in experimental transplantation [71]. In human clinical trials, the treatment of cord blood cells with dimethyl PGE2 caused an increase in long-term engraftment [72], suggesting that a compound identified in zebrafish could have clinical application in humans.

\section{B) Zebrafish as an animal model for human diseases Cardiovascular diseases}

Model fish are excellent materials for the study of human diseases due to some mutants display similar phenotypes of human diseases [73]. In addition, essential genes and thereof regulation to control the development of tissues or organs are highly conserved [74]. For example, Tbx5 is a T-box transcription factor responsible for cell-type specification and morphogenesis. The phenotypes of $t b \times 5 \mathrm{mu}-$ tant are highly similar among mammals and zebrafish. Thus, transgenic fish with heart-specific fluorescence could provide a high-through screening platform for drugs for cardiovascular disease. For example, the Tsai's lab established a transgenic line which could be induced to knock down the expression level of cardiac troponin $\mathrm{C}$ at any developmental stage, including embryos, larva or adult fish. The reduction of troponin $\mathrm{C}$ resulted in mimicry of dilated cardiomyopathy, and the incomplete atrioventricular blocking disease in humans. Therefore, this transgenic line is expected to make a significant contribution to drug screening and the elucidation of the molecular mechanisms underlying cardiovascular diseases. Next, the effect of drugs on embryonic development was also studied.

Amiodarone, which is a class III antiarrhythmic agent, is being used for the treatment of tachyarrhythmia in humans. However, Amiodarone-treated zebrafish embryos were found to exhibit backflow of blood in the heart [75]. Subsequent research showed that Amiodarone caused failure of cardiac valve formation [75]. Specifically, Amiodarone induces ectopic expression of similar to versican $b$ $(s-v c a n b)$, resulting in repression of EGFR/GSK3 $\beta /$ Snail signaling, which in turn, upregulates $c d h 5$ at the heart field, and causes defective cardiac valves [76]. Moreover, Amiodarone was found to repress metastasis of breast cancer cells by inhibiting the EGFR/ERK/Snail pathway [77], a phenomenon analogous to the inhibitory effects of Amiodarone on EMT transition observed in the heart.

Last but not least, although zebrafish has a twochambered heart, relative to mouse, rat, and rabbit, its heart rate, action potential duration (APD) and electrocardiogram (ECG) morphology are similar to those of humans. [78, 79]. Additionally, Tsai et al. demonstrated that the in vitro ECG recording of zebrafish heart is a simple, efficient and high throughput assay [80]. Thus, zebrafish can serve as a platform for direct testing of drug effect on APD prolongation and prolonged QT interval, which is required by the FDA as a precondition for drug approval.

\section{Cancer study: melanoma}

Zebrafish become a popular experimental animal for the studies of human cancer [81], in part because the fish homologs of human oncogenes and tumor suppressor genes have been identified, and in part because signaling pathways regulating cancer development are conserved [82-84]. Amatruda et al. reported that many zebrafish tumors are similar to those of human cancer in the 
histological examination [85]. The zebrafish transgenic line with skin-specific red fluorescence could be applied for skin tumor detection [86]. When the embryos of this line were treated with solutions containing arsenic, the tumors induced on the skin could be easily identified by naked eye under fluorescent microscope. Therefore, this transgenic line can be potentially used for the study of skin diseases. For example, the common skin cancer melanoma may be screened by the red fluorescence expression in this transgenic line.

Zebrafish transgenic line could also be applied to establish models simulating melanoma development. The human oncogenic BRAF V600E was expressed under the control of the zebrafish melanocyte mitfa promoter to establish a melanoma model [87]. Combining skinspecific red fluorescence with mitfa-driven oncogene expression, the melanoma could be easily traced. Therefore, transgenic lines and mutants of model fish could provide abundant resources for mechanistic studies and therapeutic research in human diseases.

\section{Cancer study: metastasis}

Metastasis involves processes of sequential, interlinked and selective steps, including invasion, intravasation, arrest in distant capillaries, extravasation, and colonization [88]. Zebrafish is again an alternative organism for in vivo cancer biology studies. In particular, xenotransplantation of human cancer cells into zebrafish embryos serves as an alternative approach for evaluating cancer progression and drug screening [89]. For example, human primary tumor cells labeled with fluorescence have already been implanted in zebrafish liver, and the invasiveness and metastasis of these cells were directly observable and easily traceable [90]. To investigate the mechanism of local cancer cell invasion, human glioblastoma cells labeled with fluorescence were infiltrated into the brain of zebrafish embryos. It was observed that the injected cells aligned along the abluminal surface of brain blood vessels [91]. By grafting a small amount of highly metastatic human breast carcinoma cells onto the pericardial membrane of zebrafish embryos at $48 \mathrm{hpf}$, tumor cells were observed to move longitudinally along the aorta [92]. Similarly, highly metastatic human cancer cells labeled with fluorescence were injected into the pericardium of 48-hpf embryos. Afterwards, it is possible to visualize how cancer cells entered the blood circulation and arrested in small vessels in head and tail [93]. In another example, zebrafish embryos were injected with tumorigenic human glioma stem cells at different stages of metastasis, including beginning, approaching, clustering, invading, migrating, and transmigrating [94]. Thus, grafting a small number of labeled tumor cells into transparent zebrafish embryos allows us to dynamically monitor the cancer cells without the interference of immune suppression.
Apart from its utility in analyzing the mechanisms of tumor dissemination and metastasis, the zebrafish model can also be applied to screen potential anticancer compounds or drugs. In addition, zebrafish feature such advantages as easy gene manipulation, short generation cycle, high reproducibility, low maintenance cost, and efficient plating of embryos $[95,96]$. Therefore, this small fish is second only to SCID and nude mice as xenograft recipients of cancer cells.

\section{Cancer study: leukemia}

Leukemia is a cancer related to hematopoiesis. Most often, leukemia results from the abnormal increase of white blood cells. However, some human cancers of bone marrow and blood origins have their parental cells from other blood cell types. The search for efficacious therapies for leukemia is ongoing. Interestingly, the developmental processes and genes related to hematopoiesis are similar between zebrafish and humans, making zebrafish a feasible model for the study of leukemia. In addition, gene expression in zebrafish could be conveniently modified by several approaches, e.g., MO-induced gene knockdown, TALENs and CRISPR/Cas9 gene knockout, and DNA/ RNA introduced overexpression [6, 7]. In the study of Yeh et al. [97], the zebrafish model was applied to screen for chemical modifiers of AML1-ETO, an oncogenic fusion protein prevalent in acute myeloid leukemia (AML). Treatment of zebrafish with chemical modifiers of AML1-ETO resulted in hematopoietic dysregulation and elicited a malignant phenotype similar to human AML. Cyclooxygenase-2 (COX2) is an enzyme causing inflammation and pain. Nimesulide is an inhibitor of COX2 and an antagonist to AML1-ETO in hematopoietic differentiation.

FMS-like tyrosine kinase 3 (FLT3) is a class III receptor tyrosine kinase which is normally expressed in human hematopoietic stem and progenitor cells (HSPCs) [98]. Internal tandem duplication (ITD), which may occur at either the juxtamembrane domain (JMD) or the tyrosine kinase domain (TKDs) of FLT3, is observed in one-third of human AML. Zebrafish Flt3 shares an overall 32, 35, and $34 \%$ sequence identity with that of human, mouse, and rat, respectively. However, the JMD and the activation loops of TKD are highly conserved, implicating that the functions of FLT3 signaling are evolutionally conserved. Overexpression of human FLT3-ITD in zebrafish embryos induces the ectopic expansion of FLT3-ITD positive myeloid cells. If those embryos are treated with AC220, a potent and relatively selective inhibitor of FLT3, FLT3-ITD myeloid expansion is effectively ameliorated [99]. In another example, isocitrate dehydrogenase (IDH) 1 and 2 are involved in citric acid cycle in intermediary metabolism. IDH mutations are found in approximately $30 \%$ of cytogenetically abnormal AML, suggesting a pathogenetic link in leukemia initiation [100, 101]. Injection of either 
human IDH1-R132H or zebrafish idh1-R146H, a mutant corresponding to human IDH1-R132H, resulted in increased 2-hydroxyglutarate, which in turn induced the expansion of primitive myelopoiesis [102]. Taken together, these reports suggest that the molecular pathways involved in leukemia are conserved between humans and zebrafish. Based on the aforementioned experimental evidence, zebrafish can be an exceptional platform for mimicking human myelodysplastic syndromes and establishing an in vivo vertebrate model for drug screening.

\section{Cancer study: hepatoma}

Several liver tumor models have been reported by liverspecific expression of transgenic oncogenes such as kras, $x m r k$ and $m y c$. These transgenic lines of zebrafish usually generate liver tumors with various severity from hepatocellular adenoma (HCA) to hepatocellular carcinoma (HCC) [103-105]. These three transgenic liver cancer models have been used to identify differentially expressed genes through RNA-SAGE sequencing. For example, researchers have searched genes either up- or downregulated among the three tumor models and analyzed the possible signaling pathways. Then, correlation between zebrafish liver tumor signatures and the different stages of human hepatocarcinogenesis was determined [106]. High tumor incidence and convenient chemical treatment make this inducible transgenic zebrafish a plausible platform for studying on liver tumor progression, regression, and anticancer drug screening.

\section{Behavioral studies}

Interestingly, zebrafish become a modern organism for studying on depressive disorders [107-109]. Because the physiological (neuroanatomical, neuroendocrine, neurochemical) and genetic characteristics of zebrafish are similar to mammals, zebrafish are ideal for high-throughput genetic and chemical genetic screening. Furthermore, since behavioral test of zebrafish for cognitive, approach-avoidance, and social paradigms are available, the identification of depression-like indices in response to physiological, genetic, environmental, and/ or psychopharmacological alterations is feasible [110]. Actually, zebrafish display highly robust phenotypes of neurobehavioral disorders such as anxiety-like and approach-avoidance behaviors. Furthermore, novel information of behavioral indices can be exposed, including geotaxis via top-bottom vertical movement [111]. Zebrafish behavior can also be monitored using automated behavioral tracking software, which enhances efficiency and reduces interrater variance [112]. Additionally, zebrafish offer a potential insight into the social aspects of depression [113] and may be suitable for studying the cognitive deficits of depression [114] and its putative etiological pathways [115]. Last but not least, zebrafish are highly sensitive to psychotropic drugs, such as antidepressants, anxiolytics, mood stabilizers, and antipsychotics [116-118], serving as an important tool for drug discovery.

\section{C) Biosensor for environmental toxicants}

Aromatic hydrocarbons, heavy metals and environmental estrogens are currently being used to test the impact of environmental pollutants on animals [119]. These studies mainly focused on mortality and abnormality rates. However, the developing embryos may have already been damaged in a subtle way that would have precluded direct observation of morphology and detection of mortality. To overcome this drawback, transgenic fish can be used because they are designed to study (a) whether toxicants cause defective genes during embryogenesis; (b) whether pollutants affect the expression of tissue-specific gene; and (c) whether the impact of pollutants on embryonic development is dosage dependent. Pollutants can be directly detected by simply observing the coloration change of cells before or after the pollutants can cause morphological damage. Therefore, transgenic model fish are promising organisms for use as bioindicators to environmental toxicants and mutagens [120, 121]. In addition, Chen and $\mathrm{Lu}$ reported that the environmental xenobiotics can be detected by a transgenic line of medaka carrying a GFP reporter driven by cytochrome P450 1a promoter (CYP1A-GFP) [122]. Furthermore, the environmental xenoestrogenic compounds can be specifically detected by a hybrid transgenic line derived from crossing between line CYP1A-GFP and line VG-Lux whose Lux reporter activity is driven by a vitellogenin promoter [123]. Lee et al. reported another zebrafish transgenic line, termed huORFZ [54], as it has been described in pervious section. At normal condition, the translation of the transferred huORF ${ }^{\text {chop }}$-gfp mRNA in huORFZ embryos is completely suppressed by an inhibitory uORF of human chop mRNA (huORF ${ }^{\text {chop }}$ ). However, when the huORFZ embryos were under ER stress, such as heat shock, cold shock, hypoxia, metals, alcohol, toxicants or drugs, the downstream GFP became apparent due to the blockage of huORF ${ }^{\text {chop }}$-mediated translation inhibition. Therefore, huORFZ embryos can be used to study the mechanism of translational inhibition. Additionally, huORFZ embryos can serve a living material to monitor the contamination of hazardous pollutants [124]. Besides the universal huORFZ system, zebrafish could also be indicators for specific pollutants. For example, Xu et al. reported a transgenic zebrafish $T g$ (cyp1a:gfp) which can serve as an in vivo assay for screening xenobiotic compounds, since Cyp1a is involved in the aryl hydrocarbon receptor pathway, and can be induced in the presence of Dioxins/Dioxin-like compounds and polycyclic aromatic hydrocarbons [125]. Additional advantages of zebrafish 
include the small size, abundant number, rapid development and transparent eggs. These features make this model fish more accessible for the studies of molecular toxicology.

\section{Conclusion}

It is increasingly clear that the transgenic fish model is a powerful biomaterial for the studies of multiple disciplines, including molecular biology, developmental biology, neurobiology, cancer biology and regenerative medicine. It provides a simple, yet effective, in vivo approach to identify regulatory DNA sequences, as well as determine gene function and molecular pathways. More importantly, an increasing number of papers have reported that (a) the defective phenotype of mutants of model fish can photocopy with known human disorders; and (b) drugs have similar effects on zebrafish and mammalian systems. Therefore, the transgenic fish model offers a useful platform for high-throughput drug screening in biomedical sciences. Additionally, it can serve as an environmental indicator for detecting pollutants in our daily lives.

Nevertheless, there are several limitations and caveats of this fish model. First, unlike mammals, fish lack the heart septation, lung, mammary gland, prostate gland and limbs, which make the fish model impossible for studies of these tissues and organs. Additionally, fish are absent of placenta so that fish embryos are directly exposed to the environment (e.g., drugs or pollutants) without involving the placenta. Second, fish are poikilothermic and usually maintained below $30{ }^{\circ} \mathrm{C}$, which may not be optimal for those mammalian agents adapted for $37{ }^{\circ} \mathrm{C}$ in evolution. Last, since the zebrafish genome is tetraploid, it is less straight forward to conduct loss-offunction studies for certain genes.

\section{Competing interests}

The authors declare that they have no competing interests.

\section{Authors' contributions}

HJT conceptualized, organized, charged and revised the content, and HJT, CYL and CYC wrote the manuscript together. All authors read and approved the final manuscript.

\section{Acknowledgments}

This work was supported by the Ministry of Science and Technology, R. O. C. (104-2321-B-715-001).

Received: 1 November 2015 Accepted: 20 January 2016

Published: 28 January 2016

\section{References}

1. Hackett PB. The molecular biology of transgenic fish. In: Hochachka PW, Mommsen TP, editors. Biochemistry and Molecular Biology of Fishes. Amsterdam: Elsevier Science; 1993. p. 207-40.

2. Hsiao CD, Hsieh FJ, Tsai HJ. Enhanced expression and stable transmission of transgenes flanked by inverted terminal repeats from adeno-associated virus in zebrafish. Dev Dyn. 2001;220:323-36.
3. Kawakami K, Shima A. Identification of the Tol2 transposase of the medaka fish Oryzias latipes that catalyzes excision of a nonautonomous Tol2 element in zebrafish Danio rerio. Gene. 1999;240:239-44.

4. Urasaki A, Morvan G, Kawakami K. Functional dissection of the Tol2 transposable element identified the minimal cis-sequence and a highly repetitive sequence in the subterminal region essential for transposition. Genetics. 2006;174:639-49.

5. Kawakami K, Takeda H, Kawakami N, Kobayashi M, Matsuda N, Mishina M. A transposon-mediated gene trap approach identifies developmentally regulated genes in zebrafish. Dev Cell. 2004;7:133-44.

6. Huang P, Xiao A, Zhou M, Zhu Z, Lin S, Zhang B. Heritable gene targeting in zebrafish using customized TALENs. Nat Biotechnol. 2011;29:699-700.

7. Hwang WY, Fu Y, Reyon D, Maeder ML, Tsai SQ, Sander JD, et al. Efficient genome editing in zebrafish using a CRISPR-Cas system. Nat Biotechnol. 2013;31:227-9.

8. Auer TO, Del Bene F. CRISPR/Cas9 and TALEN-mediated knock-in approaches in zebrafish. Methods. 2014;69:142-50.

9. Amsterdam A, Lin S, Hopkins N. The Aequorea victoria green fluorescent protein can be used as a reporter in live zebrafish embryos. Dev Biol. 1995;171:123-9.

10. Long Q, Meng A, Wang H, Jessen JR, Farrell MJ, Lin S. GATA-1 expression pattern can be recapitulated in living transgenic zebrafish using GFP reporter gene. Development. 1997;124:4105-11.

11. Higashijima S, Okamoto H, Ueno N, Hotta Y, Eguchi G. High-frequency generation of transgenic zebrafish which reliably express GFP in whole muscles or the whole body by using promoters of zebrafish origin. Dev Biol. 1997;192:289-99.

12. Kennedy BN, Vihtelic TS, Checkley L, Vaughan KT, Hyde DR. Isolation of a zebrafish rod opsin promoter to generate a transgenic zebrafish line expressing enhanced green fluorescent protein in rod photoreceptors. J Biol Chem. 2001;276:14037-43.

13. Higashijima S, Hotta $Y$, Okamoto H. Visualization of cranial motor neurons in live transgenic zebrafish expressing green fluorescent protein under the control of the islet-1 promoter/enhancer. J Neurosci. 2000;20:206-18.

14. Huang H, Vogel SS, Liu N, Melton DA, Lin S. Analysis of pancreatic development in living transgenic zebrafish embryos. Mol Cell Endocrinol. 2001;177:117-24.

15. Huang CJ, Tu CT, Hsiao CD, Hsieh FJ, Tsai HJ. Germ-line transmission of a myocardium-specific GFP transgene reveals critical regulatory elements in the cardiac myosin light chain 2 promoter of zebrafish. Dev Dyn. 2003;228:30-40.

16. Her GM, Yeh $\mathrm{YH}, \mathrm{Wu} J \mathrm{~L}$. 435-bp liver regulatory sequence in the liver fatty acid binding protein (L-FABP) gene is sufficient to modulate liver regional expression in transgenic zebrafish. Dev Dyn. 2003;227:347-56.

17. Kim Yl, Lee S, Jung SH, Kim HT, Choi JH, Lee MS, et al. Establishment of a bone-specific col10a1: GFP transgenic zebrafish. Mol Cells. 2013;36:145-50.

18. Walton EM, Cronan MR, Beerman RW, Tobin DM. The macrophage-specific promoter mfap4 allows live, long-term analysis of macrophage behavior during mycobacterial infection in zebrafish. PLoS One. 2015;10, e0138949.

19. Krøvel AV, Olsen LC. Expression of a vas:: EGFP transgene in primordial germ cells of the zebrafish. Mech Dev. 2002;116:141-50.

20. Chou CY, Horng LS, Tsai HJ. Uniform GFP-expression in transgenic medaka (Oryzias latipes) at the F0 generation. Transgenic Res. 2001;10:303-15.

21. Holden C, Bhattacharjee Y. Random samples: That special glow. Science. 2003;300:1368.

22. Dean J. Genetics: Fish that glow in Taiwan. Far Eastern Economic Review. 2003;8:38.

23. Buechner MM, Grossman L, Hamilton A. Coolest inventions 2003: Light and dark-Red fish, blue fish and Glow-in-dark fish. TIME. 2003;24:68-9.

24. Jessen JR, Meng A, McFarlane RJ, Paw BH, Zon LI, Smith GR, et al. Modification of bacterial artificial chromosomes through chi-stimulated homologous recombination and its application in zebrafish transgenesis. Proc Natl Acad Sci U S A. 1998;95:5121-6.

25. Jessen JR, Willett CE, Lin S. Artificial chromosome transgenesis reveals longdistance negative regulation of rag1 in zebrafish. Nat Genet. 1999;23:15-6.

26. Chen YH, Wang YH, Chang MY, Lin CY, Weng CW, Westerfield M, et al. Multiple upstream modules regulate zebrafish myf5 expression. BMC Dev Biol. 2007;7:1.

27. Scheer N, Campos-Ortega JA. Use of the GAL4-UAS technique for targeted gene expression in zebrafish. Mech Dev. 1999;80:153-8.

28. Walsh EC, Stainier DY. UDP-glucose dehydrogenase required for cardiac valve formation in zebrafish. Science. 2001;293:1670-3.

29. Raya A, Koth CM, Büscher D, Kawakami Y, Itoh T, Raya RM, et al. Activation of Notch signaling pathway precedes heart regeneration in zebrafish. Proc Natl Acad Sci U S A. 2003;100 Suppl 1:11889-95. 
30. Shu X, Cheng K, Patel N, Chen F, Joseph E, Tsai HJ, et al. Na, K-ATPase is essential for embryonic heart development in the zebrafish. Development. 2003;130:6165-73.

31. Forouhar AS, Liebling M, Hickerson A, Nasiraei-Moghaddam A, Tsai HJ, Hove $J R$, et al. The embryonic vertebrate heart tube is a dynamic suction pump. Science. 2006:312:751-3.

32. Liebling M, Forouhar AS, Gharib M, Fraser SE, Dickinson ME. Four-dimensional cardiac imaging in living embryos via postacquisition synchronization of nongated slice sequences. J Biomed Opt. 2005;10:054001.

33. Hami D, Grimes AC, Tsai HJ, Kirby ML. Zebrafish cardiac development requires a conserved secondary heart field. Development. 2011;138:2389-98.

34. Nevis K, Obregon P, Walsh C, Guner-Ataman B, Burns CG, Burns CE. Tbx1 is required for second heart field proliferation in zebrafish. Dev Dyn. 2013;242:550-9.

35. Chen YH, Lee HC, Liu CF, Lin CY, Tsai HJ. Novel regulatory sequence - 82/62 functions as a key element to drive the somite-specificity of zebrafish myf-5. Dev Dyn. 2003;228:41-50.

36. Lee HC, Huang HY, Lin CY, Chen YH, Tsai HJ. Foxd3 mediates zebrafish myf5 expression during early somitogenesis. Dev Biol. 2006;290:359-72.

37. Lee HC, Tseng WA, Lo FY, Liu TM, Tsai HJ. FoxD5 mediates anterior-posterior polarity through upstream modulator Fgf signaling during zebrafish somitogenesis. Dev Biol. 2009;336:232-45.

38. Wang YH, Li CK, Lee GH, Tsay HJ, Tsai HJ, Chen YH. Inactivation of zebrafish mrf4 leads to myofibril misalignment and motor axon growth disorganization. Dev Dyn. 2008;237:1043-50.

39. Lin CY, Chen YH, Lee HC, Tsai HJ. Novel cis-element in intron 1 represses somite expression of zebrafish myf-5. Gene. 2004;334:63-72.

40. Lin CY, Chen JS, Loo MR, Hsiao CC, Chang WY, Tsai HJ. MicroRNA-3906 regulates fast muscle differentiation through modulating the target gene homer-1b in zebrafish embryos. PLoS One. 2013;8:e70187.

41. Hsu RJ, Lin CY, Hoi HS, Zheng SK, Lin CC, Tsai HJ. Novel intronic microRNA represses zebrafish myf5 promoter activity through silencing dickkopf-3 gene. Nucleic Acids Res. 2010;38:4384-93.

42. Fu CY, Su YF, Lee MH, Chang GD, Tsai HJ. Zebrafish Dkk3a protein regulates the activity of myf5 promoter through interaction with membrane receptor integrin a6b. J Biol Chem. 2012;287:40031-42.

43. Hsu RJ, Lin CC, Su YF, Tsai HJ. dickkopf-3-related gene regulates the expression of zebrafish myf5 gene through phosphorylated p38adependent Smad4 activity. J Biol Chem. 2011;286:6855-64.

44. Lin CY, Yung RF, Lee HC, Chen WT, Chen YH, Tsai HJ. Myogenic regulatory factors Myf5 and Myod function distinctly during craniofacial myogenesis of zebrafish. Dev Biol. 2006;299:594-608.

45. Lin CY, Chen WT, Lee HC, Yang PH, Yang HJ, Tsai HJ. The transcription factor Six1a plays an essential role in the craniofacial myogenesis of zebrafish. Dev Biol. 2009;331:152-66.

46. Lin CY, Lee HC, Chen HC, Hsieh CC, Tsai HJ. Normal function of Myf5 during gastrulation is required for pharyngeal arch cartilage development in zebrafish embryos. Zebrafish. 2013;10:486-99.

47. Hinits Y, Osborn DP, Hughes SM. Differential requirements for myogenic regulatory factors distinguish medial and lateral somitic, cranial and fin muscle fibre populations. Development. 2009;136:403-14.

48. Ma GC, Wang TM, Su CY, Wang YL, Chen S, Tsai HJ. Retina-specific ciselements and binding nuclear proteins of carp rhodopsin gene. FEBS Lett. 2001;508:265-71

49. Hu CY, Yang CH, Chen WY, Huang CJ, Huang HY, Chen MS, et al. Egr1 gene knockdown affects embryonic ocular development in zebrafish. Mol Vis. 2006;12:1250-8.

50. Huang HY, Dai ES, Liu JT, Tu CT, Yang TC, Tsai HJ. The embryonic expression patterns and the knockdown phenotypes of zebrafish ADP-ribosylation factor-like 6 interacting protein gene. Dev Dyn. 2009;238:232-40.

51. Huang HY, Liu JT, Yan HY, Tsai HJ. Arl6ip1 plays a role in proliferation during zebrafish retinogenesis. Cells Tissues Organs. 2012;196:161-74.

52. Tu CT, Yang TC, Huang HY, Tsai HJ. Zebrafish arl6ip1 is required for neural crest development during embryogenesis. PLoS One. 2012;7:e32899.

53. Lin CY, Huang HY, Lu PN, Lin CW, Lu KM, Tsai HJ. Ras-related nuclea protein is required for late developmental stages of retinal cells in zebrafish eyes. Int J Dev Biol 2015;59:435-42.

54. Lee HC, Chen YJ, Liu YW, Lin KY, Chen SW, Lin CY, et al. Transgenic zebrafish model to study translational control mediated by upstream open reading frame of human chop gene. Nucleic Acids Res. 2011;39:e139.

55. Djuranovic S, Nahvi A, Green R. A parsimonious model for gene regulation by miRNAs. Science. 2011;331:550-3.
56. Huntzinger $E$, Izaurralde E. Gene silencing by microRNAs: contributions of translational repression and mRNA decay. Nat Rev Genet. 2011:12:99-110.

57. Fabian MR, Sonenberg N, Filipowicz W. Regulation of mRNA translation and stability by microRNAs. Annu Rev Biochem. 2010;79:351-79.

58. Bartel DP. MicroRNAs: target recognition and regulatory functions. Cell. 2009:136:215-33.

59. Rajewsky N. microRNA target predictions in animals. Nat Genet. 2006;38:S8-S13.

60. Hsu RJ, Yang HJ, Tsai HJ. Labeled microRNA pull-down assay system: an experimental approach for high-throughput identification of microRNAtarget mRNAs. Nucleic Acids Res. 2009;37:e77.

61. Stahlhut C, Suárez Y, Lu J, Mishima Y, Giraldez AJ. miR-1 and miR-206 regulate angiogenesis by modulating VegfA expression in zebrafish. Development. 2012;139:4356-64.

62. Lin CY, Lee HC, Fu CY, Ding YY, Chen JS, Lee MH, et al. MiR-1 and miR-206 target different genes to have opposing roles during angiogenesis in zebrafish embryos. Nat Commun. 2013;4:2829.

63. Frith JE, Porrello ER, Cooper-White JJ. Concise review: new frontiers in microRNA-based tissue regeneration. Stem Cells Transl Med. 2014;3:969-76.

64. Ujigo S, Kamei N, Hadoush H, Fujioka Y, Miyaki S, Nakasa T, et al. Administration of microRNA-210 promotes spinal cord regeneration in mice. Spine (Phila Pa 1976). 2014;39:1099-107.

65. Porrello ER, Mahmoud Al, Simpson E, Johnson BA, Grinsfelder D, Canseco D, et al. Regulation of neonatal and adult mammalian heart regeneration by the miR-15 family. Proc Natl Acad Sci U S A. 2013;110:187-92.

66. Fuller-Carter PI, Carter KW, Anderson D, Harvey AR, Giles KM, Rodger J. Integrated analyses of zebrafish miRNA and mRNA expression profiles identify miR-29b and miR-223 as potential regulators of optic nerve regeneration. BMC Genomics. 2015;16:591.

67. Traver D, Paw BH, Poss KD, Penberthy WT, Lin S, Zon LI. Transplantation and in vivo imaging of multilineage engraftment in zebrafish bloodless mutants. Nat Immunol. 2003:4:1238-46.

68. Orkin SH, Zon LI. Hematopoiesis: an evolving paradigm for stem cell biology. Cell. 2008;132:631-44.

69. Hsia N, Zon LI. Transcriptional regulation of hematopoietic stem cell development in zebrafish. Exp Hematol. 2005;33:1007-14.

70. Weber GJ, Choe SE, Dooley KA, Paffett-Lugassy NN, Zhou Y, Zon LI. Mutantspecific gene programs in the zebrafish. Blood. 2005;106:521-30.

71. North TE, Goessling W, Walkley CR, Lengerke C, Kopani KR, Lord AM, et al. Prostaglandin E2 regulates vertebrate haematopoietic stem cell homeostasis. Nature. 2007:447:1007-11.

72. Goessling W, Allen RS, Guan X, Jin P, Uchida N, Dovey M, et al. Prostaglandin E2 enhances human cord blood stem cell xenotransplants and shows long-term safety in preclinical nonhuman primate transplant models. Cell Stem Cell. 2011:8:445-58.

73. Shin JT, Fishman MC. From Zebrafish to human: modular medical models. Annu Rev Genomics Hum Genet. 2002;3:311-40.

74. Garrity DM, Childs S, Fishman MC. The heartstrings mutation in zebrafish causes heart/fin Tbx5 deficiency syndrome. Development. 2002;129:4635-45.

75. Chen YH, Lee HC, Hsu RJ, Chen TY, Huang YK, Lo HC, et al. The toxic effect of Amiodarone on valve formation in the developing heart of zebrafish embryos. Reprod Toxicol. 2012;33:233-44.

76. Lee HC, Lo HC, Lo DM, Su MY, Hu JR, Wu CC, et al. Amiodarone induces overexpression of similar to versican b to repress the EGFR/Gsk3b/Snail signaling axis during cardiac valve formation of zebrafish embryos. PloS One 2015;10:e0144751.

77. Lee HC, Su MY, Lo HC, Wu CC, Hu JR, Lo DM, et al. Cancer metastasis and EGFR signaling is suppressed by Amiodarone-induced Versican V2. Oncotarget 2015;6:42976-87.

78. Milan DJ, Jones IL, Ellinor PT, MacRae CA. In vivo recording of adult zebrafish electrocardiogram and assessment of drug-induced QT prolongation. Am J Physiol Heart Circ Physiol. 2006;291:H269-73.

79. Arnaout R, Ferrer T, Huisken J, Spitzer K, Stainier DY, Tristani-Firouzi M, et al. Zebrafish model for human long QT syndrome. Proc Natl Acad Sci U S A. 2007;104:11316-21

80. Tsai CT, Wu CK, Chiang FT, Tseng CD, Lee JK, Yu CC, et al. In-vitro recording of adult zebrafish heart electrocardiogram - a platform for pharmacological testing. Clin Chim Acta. 2011;412:1963-7.

81. Lu JW, Hsia Y, Tu HC, Hsiao YC, Yang WY, Wang HD, et al. Liver development and cancer formation in zebrafish. Birth Defects Res C Embryo Today. 2011;93:157-72.

82. Feitsma H, Cuppen E. Zebrafish as a cancer model. Mol Cancer Res. 2008;6:685-94. 
83. Payne , Look T. Zebrafish modelling of leukaemias. $\mathrm{Br} J$ Haematol. 2009;146:247-56.

84. Stoletov K, Klemke R. Catch of the day: zebrafish as a human cancer model. Oncogene. 2008:27:4509-20.

85. Amatruda JF, Shepard JL, Stern HM, Zon LI. Zebrafish as a cancer model system. Cancer Cell. 2002;1:229-31.

86. Wang YH, Chen YH, Wu TN, Lin YJ, Tsai HJ. A keratin 18 transgenic zebrafish $\mathrm{Tg}(\mathrm{k} 18(2.9): \mathrm{RFP})$ treated with inorganic arsenite reveals visible overproliferation of epithelial cells. Toxicol Lett. 2006;163:191-7.

87. Patton EE, Widlund HR, Kutok JL, Kopani KR, Amatruda JF, Murphey RD, et al. BRAF mutations are sufficient to promote nevi formation and cooperate with p53 in the genesis of melanoma. Curr Biol. 2005;15:249-54.

88. Fidler IJ. The pathogenesis of cancer metastasis: the 'seed and soil' hypothesis revisited. Nat Rev Cancer. 2003;3:453-8.

89. Zhang B, Xuan C, Ji Y, Zhang W, Wang D. Zebrafish xenotransplantation as a tool for in vivo cancer study. Fam Cancer. 2015;14:487-93.

90. Marques IJ, Weiss FU, Vlecken DH, Nitsche C, Bakkers J, Lagendijk AK, et al. Metastatic behaviour of primary human tumours in a zebrafish xenotransplantation model. BMC Cancer. 2009:9:128.

91. Lal S, La Du J, Tanguay RL, Greenwood JA. Calpain 2 is required for the invasion of glioblastoma cells in the zebrafish brain microenvironment. J Neurosci Res. 2012;90:769-81.

92. Zhao $C$, Yang $H$, Shi $H$, Wang $X$, Chen $X$, Yuan $Y$, et al. Distinct contributions of angiogenesis and vascular co-option during the initiation of primary microtumors and micrometastases. Carcinogenesis. 2011;32:1143-50.

93. Stoletov K, Kato H, Zardouzian E, Kelber J, Yang J, Shattil S, et al. Visualizing extravasation dynamics of metastatic tumor cells. J Cell Sci. 2010;123:2332-41.

94. Yang XJ, Cui W, Gu A, Xu C, Yu SC, Li TT, et al. A novel zebrafish xenotransplantation model for study of glioma stem cell invasion. PLoS One. 2013;8:e61801.

95. Zhang B, Shimada Y, Kuroyanagi J, Umemoto N, Nishimura Y, Tanaka T. Quantitative phenotyping-based in vivo chemical screening in a zebrafish model of leukemia stem cell xenotransplantation. PLoS One. 2014;9:e85439.

96. Shimada Y, Nishimura Y, Tanaka T. Zebrafish-based systems pharmacology of cancer metastasis. Methods Mol Biol. 2014;1165:223-38.

97. Yeh JR, Munson KM, Elagib KE, Goldfarb AN, Sweetser DA, Peterson RT. Discovering chemical modifiers of oncogene-regulated hematopoietic differentiation. Nat Chem Biol. 2009;5:236-43.

98. Small D, Levenstein M, Kim E, Carow C, Amin S, Rockwell P, et al. STK-1, the human homolog of Flk-2/Flt-3, is selectively expressed in CD34+ human bone marrow cells and is involved in the proliferation of early progenitor/ stem cells. Proc Natl Acad Sci U S A. 1994;91:459-63.

99. He BL, Shi X, Man CH, Ma AC, Ekker SC, Chow HC, et al. Functions of flt3 in zebrafish hematopoiesis and its relevance to human acute myeloid leukemia. Blood. 2014;123:2518-29.

100. Gross S, Cairns RA, Minden MD, Driggers EM, Bittinger MA, Jang HG, et al. Cancer-associated metabolite 2-hydroxyglutarate accumulates in acute myelogenous leukemia with isocitrate dehydrogenase 1 and 2 mutations. J Exp Med. 2010;207:339-44.

101. Cairns RA, Harris IS, Mak TW. Regulation of cancer cell metabolism. Nat Rev Cancer. 2011;11:85-95.

102. Shi $X$, He BL, Ma AC, Guo Y, Chi Y, Man CH, et al. Functions of idh1 and its mutation in the regulation of developmental hematopoiesis in zebrafish. Blood. 2015;125:2974-84.

103. Li Z, Huang X, Zhan H, Zeng Z, Li C, Spitsbergen JM, et al. Inducible and repressable oncogene-addicted hepatocellular carcinoma in Tet-on xmrk transgenic zebrafish. J Hepatol. 2012;56:419-25.

104. Nguyen AT, Emelyanov A, Koh CH, Spitsbergen JM, Parinov S, Gong Z. An inducible kras (V12) transgenic zebrafish model for liver tumorigenesis and chemical drug screening. Dis Model Mech. 2012;5:63-72.

105. Li Z, Zheng W, Wang Z, Zeng Z, Zhan H, Li C, et al. A transgenic zebrafish liver tumor model with inducible Myc expression reveals conserved Myc signatures with mammalian liver tumors. Dis Model Mech. 2013;6:414-23.

106. Zheng W, Li Z, Nguyen AT, Li C, Emelyanov A, Gong Z. Xmrk, kras and myc transgenic zebrafish liver cancer models share molecular signatures with subsets of human hepatocellular carcinoma. PLoS One. 2014;9, e91179.

107. Kalueff AV, Echevarria DJ, Stewart AM. Gaining translational momentum: more zebrafish models for neuroscience research. Prog Neuropsychopharmacol Biol Psychiatry. 2014;55:1-6.

108. Kalueff AV, Stewart AM, Gerlai R. Zebrafish as an emerging model for studying complex brain disorders. Trends Pharmacol Sci. 2014;35:63-75.
109. Stewart AM, Braubach O, Spitsbergen J, Gerlai R, Kalueff AV. Zebrafish models for translational neuroscience research: from tank to bedside. Trends Neurosci. 2014;37:264-78.

110. Fonseka TM, Wen XY, Foster JA, Kennedy SH. Zebrafish models of major depressive disorders. J Neurosci Res. 2016;94:3-14.

111. Cachat J, Stewart A, Utterback E, Hart P, Gaikwad S, Wong K, et al. Threedimensional neurophenotyping of adult zebrafish behavior. PLoS One. 2011; 6:e17597.

112. Nguyen M, Stewart AM, Kalueff AV. Aquatic blues: modeling depression and antidepressant action in zebrafish. Prog Neuropsychopharmacol Biol Psychiatry. 2014:55:26-39.

113. Teles MC, Dahlbom SJ, Winberg S, Oliveira RF. Social modulation of brain monoamine levels in zebrafish. Behav Brain Res. 2013;253:17-24.

114. Karnik I, Gerlai R. Can zebrafish learn spatial tasks? An empirical analysis of place and single CS-US associative learning. Behav Brain Res. 2012;233:415-21.

115. Carvalho AF, Miskowiak KK, Hyphantis TN, Kohler CA, Alves GS, Bortolato B, et al. Cognitive dysfunction in depression-pathophysiology and novel targets. CNS Neurol Disord Drug Targets. 2014;13:1819-35.

116. Ellis LD, Soanes KH. A larval zebrafish model of bipolar disorder as a screening platform for neuro-therapeutics. Behav Brain Res. 2012;233:450-7.

117. Maximino C, Puty B, Benzecry R, Araújo J, Lima MG, De Jesus Oliveira Batista $E_{\text {, et }}$ al. Role of serotonin in zebrafish (Danio rerio) anxiety: relationship with serotonin levels and effect of buspirone, WAY 100635, SB 224289, fluoxetine and para-chlorophenylalanine (pCPA) in two behavioral models. Neuropharmacology. 2013;71:83-97.

118. Ziv L, Muto A, Schoonheim PJ, Meijsing SH, Strasser D, Ingraham HA, et al. An affective disorder in zebrafish with mutation of the glucocorticoid receptor. Mol Psychiatry. 2013;18:681-91.

119. Schwarzenbach RP, Egli T, Hofstetter TB, Von Gunten U, Wehrli B. Global Water Pollution and Human Health. Annu Rev Env Resour. 2010;35:109-36.

120. Amanuma $\mathrm{K}$, Takeda H, Amanuma H, Aoki Y. Transgenic zebrafish for detecting mutations caused by compounds in aquatic environments. Nat Biotechnol. 2000;18:62-5.

121. Amanuma K, Tone S, Saito H, Shigeoka T, Aoki Y. Mutational spectra of benzo [a] pyrene and MelQx in rpsL transgenic zebrafish embryos. Mutat Res. 2002;513:83-92.

122. Chen T, Lu JK. Transgenic fish technology: basic principles and their application in basic and applied research. In: De la Fuente J, Castro FO, editors. Gene Transfer in Aquatic Organism. Berlin: Springer; 1998; p. 45-73.

123. Ng GH, Gong Z. GFP transgenic medaka (Oryzias latipes) under the inducible cypla promoter provide a sensitive and convenient biological indicator for the presence of TCDD and other persistent organic chemicals. PLoS One. 2013;8:e64334.

124. Lee HC, Lu PN, Huang HL, Chu C, Li HP, Tsai HJ. Zebrafish transgenic line huORFZ is an effective living bioindicator for detecting environmental toxicants. PLoS One. 2014:9:e90160.

125. Xu H, Li C, Li Y, Ng GH, Liu C, Zhang X, et al. Generation of Tg (cypla:gfp) transgenic zebrafish for development of a convenient and sensitive in vivo assay for aryl hydrocarbon receptor activity. Mar Biotechnol (NY). 2015;17:831-40.

\section{Submit your next manuscript to BioMed Central and we will help you at every step:}

- We accept pre-submission inquiries

- Our selector tool helps you to find the most relevant journal

- We provide round the clock customer support

- Convenient online submission

- Thorough peer review

- Inclusion in PubMed and all major indexing services

- Maximum visibility for your research

Submit your manuscript at www.biomedcentral.com/submit 